\title{
Research of the Self-Restoration Speed of Low Remanence Toroid Cores
}

\author{
Z. HUANG AND L. ZHANG \\ Institute of Fluid Physics, CAEP, P.O. Box 919-106, Mianyang, Sichuan, 621900, P.R. China
}

\begin{abstract}
Theory analysis and experiments have been done to find out the factors which decide on the self-restoration speed of low remanence toroid cores. The results show that in the right working circuit, the core with appropriate pulse permeability can be restored to its remanence after excitation within $1 \mu$ s without reset current. Therefore, the toroid core restoration in a $\mathrm{MHz}$ repeat frequency, multi-pulse induction cavity is likely to be solved by choosing the right material of cores and use proper working circuit.
\end{abstract}

PACS numbers: 01.30.Cc, 75.60.Ej

\section{Introduction}

Generally, the toroid cores are used to generate inductive pulses in a pulsed power system. In many systems, the inductive devices generate several high voltage pulses with the toroid cores working in $\mathrm{MHz}$ repeating rates, such as the acceleration cells of an Induction Synchrotron [1], and a multi-pulse Linear Inductive Accelerator (LIA) [2] and the cells of an Inductive-add Kicker Modulator [3]. Therefore these toroid cores require a very fast reset before each pulse [4], otherwise they will saturate during a voltage pulse [5].

When the drive pulse is off, the magnetic flux of the toroid core will restore to its remanence flux $\left(B_{\mathrm{r}}\right)$ automatically. That means, to a toroid core, the flux between the $B_{\mathrm{r}}$ and $B_{\mathrm{s}}$ (saturation flux) can be used repeatedly without any reset current. So that if the core's $B_{\mathrm{r}}$ is close to zero and time of the core's self-restoration from $B_{\mathrm{s}}$ to $B_{\mathrm{r}}$ is less than $1 \mu \mathrm{s}$, the toroid core can generate
$\mathrm{MHz}$ frequency inductive pulses without additional reset system.

\section{What determines the self-restoration speed of low $B_{\mathrm{r}}$ core?}

Firstly, during the drive pulse on, the loop around the toroid cores play as a load of the pulsed power system, and part of the power will be stored in the cores when the current passes the loop. As the drive pulse is off, the power system cuts off from the loop, but the current will keep a while for the inductance of the loop. The power which is stored in the cores before will release, the loop plays as a power supply then. Therefore, when the drive pulse is off, increase in the resistance of the load of cores' loop can increase the restore voltage on the cores, and speed on the toroid cores' self-restoration.

TABLE I

Parameter's comparison of the low remanence toroid cores.

\begin{tabular}{c|c|c|c|c|c|c|c|c}
\hline \hline \multirow{2}{*}{ No. } & \multirow{2}{*}{ Material } & \multicolumn{2}{|c|}{$50 \mathrm{~Hz}$} & $1 \mathrm{MHz}$ & $2 \mathrm{MHz}$ & $3 \mathrm{MHz}$ & $4 \mathrm{MHz}$ \\
\cline { 3 - 8 } & & $B_{\mathrm{r}} / B_{\mathrm{s}}[\mathrm{T}]$ & $\mu_{\mathrm{r}}$ & \multicolumn{4}{|c}{$\mu_{\mathrm{i}}$} \\
\hline core A & nanocrystallization & $0.13 / 1.5$ & 2200 & 1800 & 1080 & 750 & 580 \\
core B & amorphous & $0.15 / 1.2$ & 40000 & 2300 & 1500 & 1130 & 930 \\
core C & amorphous & $0.11 / 1.5$ & 1800 & 1200 & 800 & 570 & 440 \\
core D & amorphous & $0.10 / 1.3$ & 1000 & 800 & 560 & 320 & 260
\end{tabular}

Secondly, the remanence flux $B_{\mathrm{r}}$ is the magnetic flux when the current passing the core's loop attenuates to zero. So, speed on the current attenuation after the drive pulse can short the restoration time of the core, and that requires the inductance of the toroid cores' loop as low as enough. Therefore, the decrease in the cores' permeability $\mu$ in short pulse can speed on the toroid cores' self-restoration.

In order to examine the analysis above, four low remanence toroid cores were chosen to make experiments. 
The properties of the cores are shown in Table I, $\mu_{\mathrm{i}}$ is the initialization permeability, $\mu_{\mathrm{r}}$ is the average permeability in $50 \mathrm{~Hz} \mathrm{AC}$.

\section{Effect of working circuit to self-restoration speed}

Three drive circuits were designed in this part. Sample "core A", the nanocrystallization core, was taken into these circuits.

Figure 1a shows the circuit 1 which simulates an acceleration module in LIA. A $50 \Omega$ cable with 60 ns length connects the pulsed power system and the loop around the toroid core. Parallel to the loop, there is a $50 \Omega$ resistance to match the cable. In the beginning $120 \mathrm{~ns}$ after the drive pulse off, the loop played as a power supply, then the resistance of the core's loop is $25 \Omega$ (50 $\Omega$ cable parallel connects $50 \Omega$ resistance); after that, the load should consider the $3 \mu \mathrm{F}$ capacitance. Figure $1 \mathrm{~b}, \mathrm{c}$ shows the waveform and the $B-T$ curve. Obviously, during the beginning $120 \mathrm{~ns}$ (380-500 ns in Fig. 1c), the magnetic flux $B$ reduces soon, and then it slows down as the resistance changes.

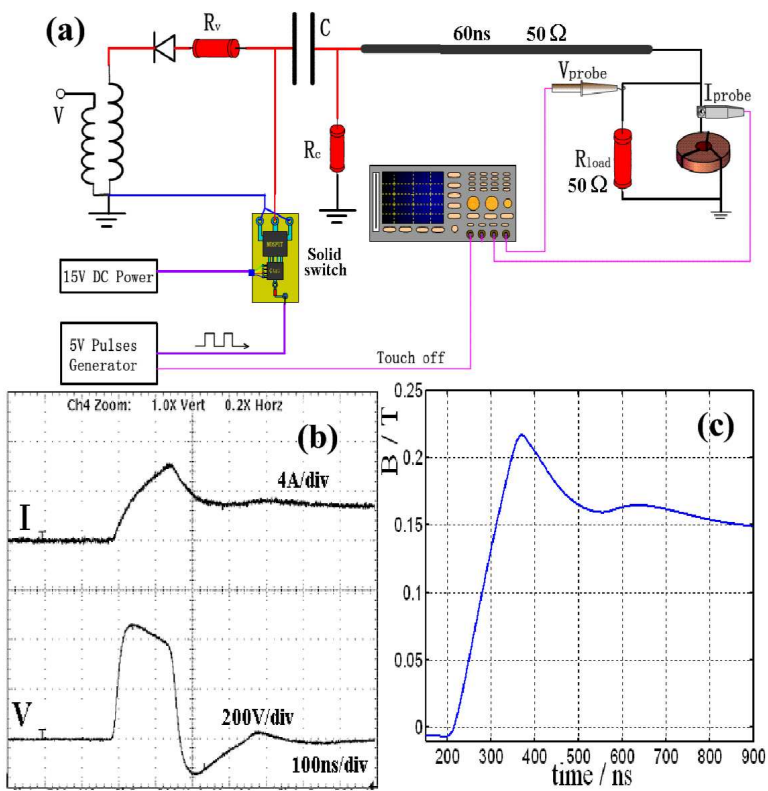

Fig. 1. (a) The experiment circuit 1, (b) the voltage and current waveform of the winding, (c) the $B-T$ curve of core A.

The time of the core A self-restoration to $B_{\mathrm{r}}$ in this circuit is more than $1 \mu \mathrm{s}$.

In circuit 2 , the length of the cable was extended to $1000 \mathrm{~ns}$ and took out the resistance which was parallel connected to the core's loop. Then, the resistance of the loop would change to $50 \Omega$ during $2 \mu$ s after drive pulse off. As Fig. 2c shows, core A can get back to $B_{\mathrm{r}}$ in $1 \mu \mathrm{s}$ in this circuit.

In the third experiment, a capacitance of $20 \mu \mathrm{F}$ displaced the $3 \mu \mathrm{F}$ capacitance. In place of the loop of the

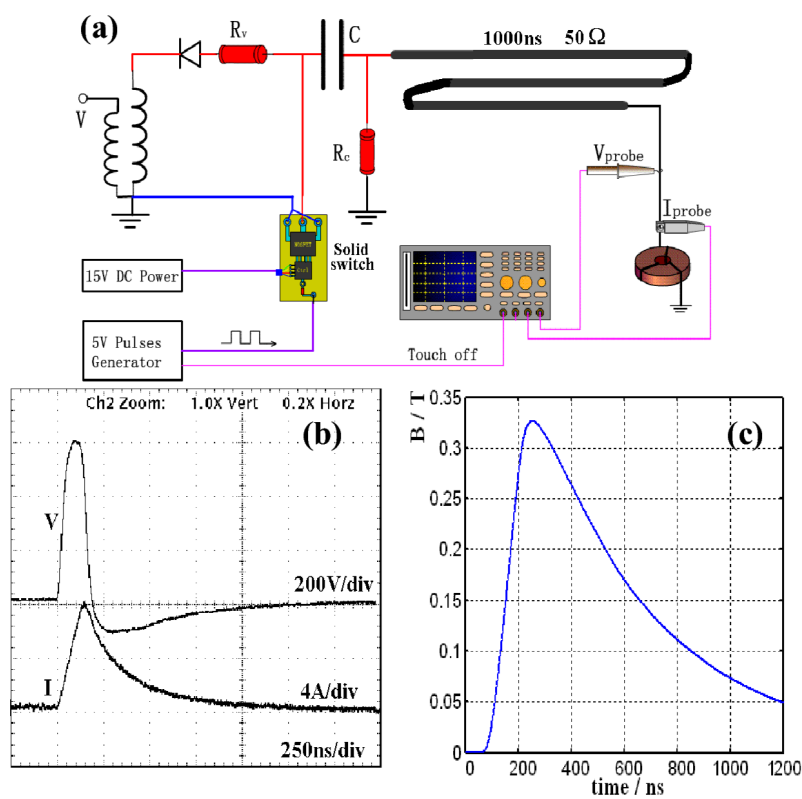

Fig. 2. (a) The experiment circuit 2, (b) the voltage and current waveform of the winding, (c) the $B-T$ curve of core $\mathrm{A}$.
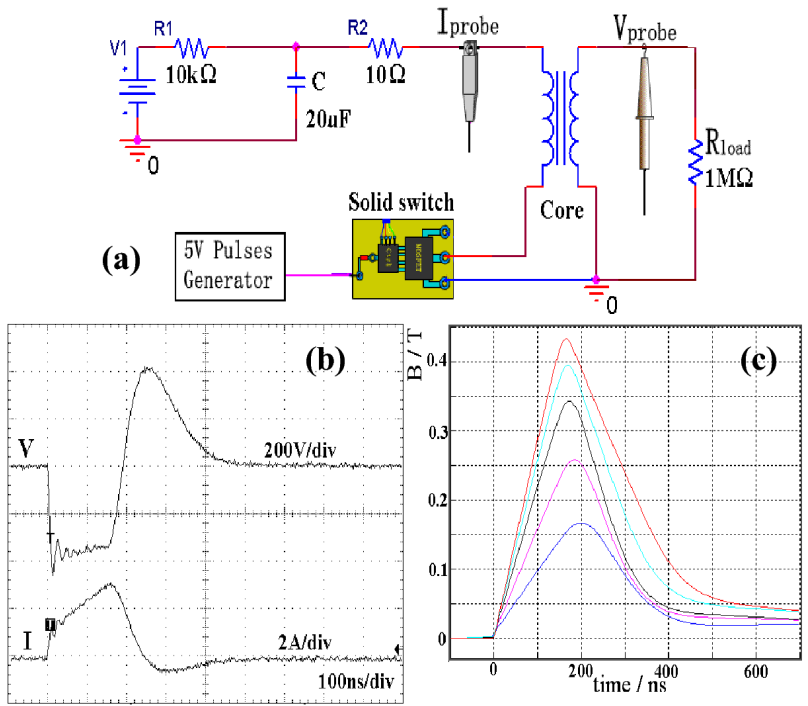

Fig. 3. (a) The experiment circuit 3, (b) the voltage and current waveform of the winding, (c) the $B-T$ curve of core A.

core, the MOSFET switch and the capacitance was connected in series, as Fig. 3 shows. So when the drive pulse is off, because the switch opened, the resistance of the core's loop is very big. Figure $3 \mathrm{~b}$ shows the current reduced fastly. As Fig. 3c shows, core A can restore itself to $B_{\mathrm{r}}$ in $300 \mathrm{~ns}$ independent of the voltage of drive pulse.

To be a conclusion, the increase in the load resistance of the cores' loop from the drive circuit can speed up the toroid cores' self-restoration. 


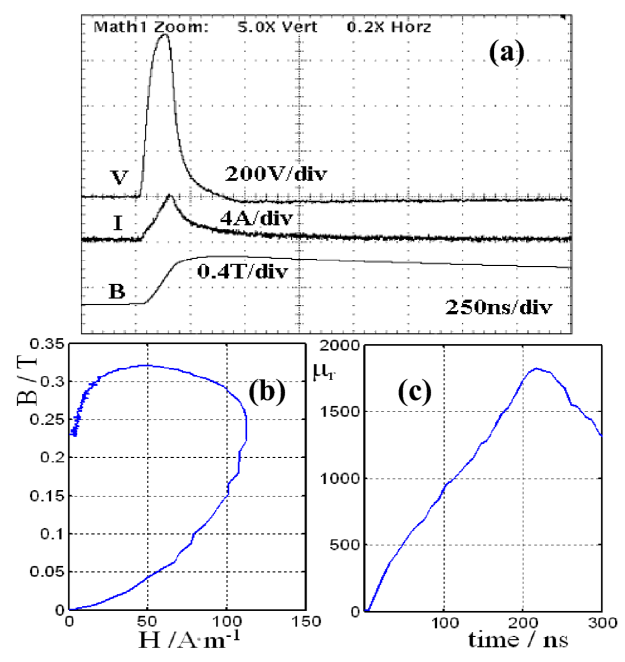

Fig. 4. The voltage, current, $B-T$ (a), magnetization (b), permeability (c) curve of core B.

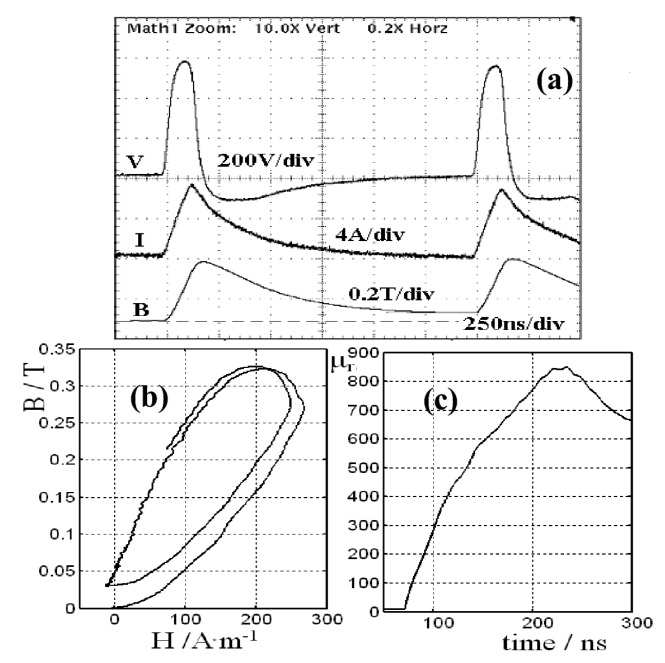

Fig. 5. The voltage, current, $B-T$ (a), magnetization (b), permeability (c) curve of core C.

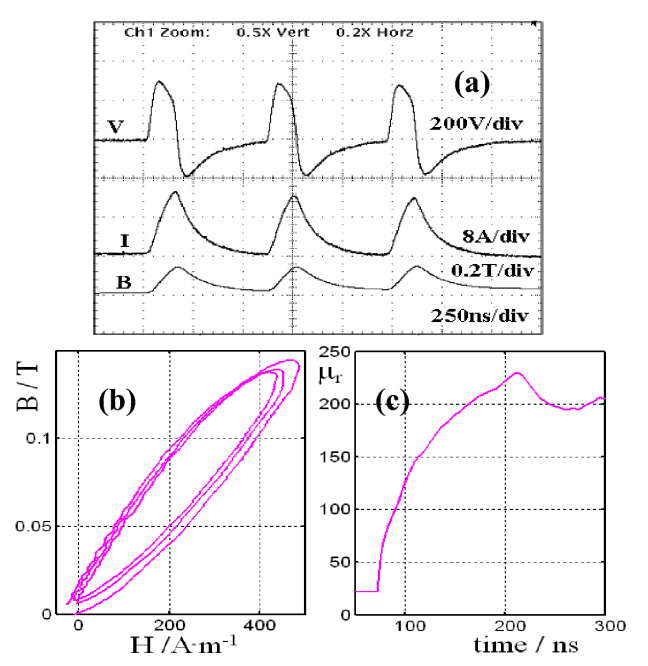

Fig. 6. The voltage, current, $B-T$ (a), magnetization (b), permeability (c) curve of core D.

\section{Effect of core's permeability to self-restoration speed}

Let us take the second circuit to test the self-restoration speed of cores B, C, D. Figures 4-6 show the examining results of the toroid cores. Table II shows the pulse average $\mu_{\mathrm{r}}$ and the self-restoration time of each core in this circuit.

TABLE II

The experiment results of the different cores in circuit B.

\begin{tabular}{c|c|c|c}
\hline \hline No. & \multicolumn{2}{|c|}{$\begin{array}{r}\text { Maximal } \mu_{\mathrm{r}} \\
\text { in pulse excitation }\end{array}$} & $\begin{array}{c}\text { Average } \mu_{\mathrm{r}} \\
\text { time }\end{array}$ \\
\hline core A & 900 & 450 & $<1 \mu \mathrm{s}$ \\
core B & 1800 & 900 & $>2 \mu \mathrm{s}$ \\
core C & 850 & 450 & $<1 \mu \mathrm{s}$ \\
core D & 230 & 150 & $<0.5 \mu \mathrm{s}$
\end{tabular}

The results appear that the decrease in the cores' permeability $\mu_{\mathrm{r}}$ in short pulses can speed on the toroid cores' self-restoration. If the pulse average permeability $[6]$ is about 400 (as core A and core $\mathrm{C}$ ), the self-restoration time is less than $1 \mu \mathrm{s}$ in circuit 2 .

Decreasing the core's pulse permeability, such as core $\mathrm{D}$, the core's self-restoration time can be shortened to $0.5 \mu \mathrm{s}$. But considering the quality of the inductive pulses, the toroid core needs enough permeability, for example, to keep a $100 \mathrm{~ns}$ flat top of the inductive pulse, and the core's average relative permeability $\mu_{\mathrm{r}}$ should be higher than 400 . Therefore, to satisfy both the requirements of inductive pulse waveform and self-restoration speed, the toroid core's average pulse $\mu_{\mathrm{r}}$ should be close to 400 .

\section{Conclusion}

The paper analyses the factors which can determine the self-restoration speed of the toroid core. The experiments proved that choosing appropriate working circuit and appropriate low permeability core, the magnetic cores' restoration problem in $\mathrm{MHz}$ frequency pulse excitation can be solved by the core's self-restoration ability.

\section{References}

[1] K. Takayama, J. Kishiro, Nucl. Instrum. Methods Phys. Res. A 451, 304 (2000).

[2] S.F. Chen, J.J. Deng, Nucl. Instrum. Methods Phys. Res. A 579, 941 (2007).

[3] E. Cook, B. Lee, in: Proc. XX Int. Linac Conf., Monterey, California 2000.

[4] Z.P. Huang, L. Zhang, in: Proc. First Euro-Asian Pulsed Power Conf. 2006, p. 173.

[5] R.C. O'Handley, Modern Magnetic Materials Principle and Applications, Chemistry Industry Press, Beijing 2002.

[6] Z.P. Huang, L. Qin, High Power Laser Part. Beams 19, 125 (2007). 